\title{
Rotary and displacement tuners for multistub cavities
}

\author{
N. R. Lobanov and D. C. Weisser \\ Department of Nuclear Physics, Research School of Physical Sciences and Engineering, Australian National University, \\ Canberra, ACT 0200, Australia
}

(Received 26 February 2007; published 20 June 2007)

\begin{abstract}
Rotary and displacement tuners are described for multistub superconducting rf resonators. The effectiveness of these tuners is made possible because the resonators have low currents between their outer conductors and tuner elements. Computer simulations and experimental data show that the devices provide a tuning range up to $100 \mathrm{kHz}$ with a frequency resolution of about $1 \mathrm{~Hz}$. As well, only a small driving force is required allowing use of a low-backlash drive mechanism. The use of the rotary tuner is limited to the resonators with two loading elements such as the 2-stub quarter wave resonator, the conventional split loop resonator, and the 2-stub, half wave resonator. The displacement tuner is more versatile and can be used for any TEM-like quarter wave resonator or half wave resonator resonators with two or more loading elements.
\end{abstract}

DOI: 10.1103/PhysRevSTAB.10.062001

PACS numbers: 29.17.+w, 29.27. $-\mathrm{a}, 41.75 .-\mathrm{i}$

\section{INTRODUCTION}

The electromagnetic and mechanical properties of $150 \mathrm{MHz}, \lambda / 4$ superconducting accelerating structures, with multiple loading elements, are described in $[1,2]$. Figure 1 shows the prototype 2 -stub quarter wave resonator (2-QWR) halfway through the computer numerical control (CNC) machining process.

The tuner has to be able to adjust the resonator frequency to within the range of the phase feedback system, typically $100 \mathrm{~Hz}$ out of $150 \mathrm{MHz}$. Since the frequency shift upon cooldown is $\sim 500 \mathrm{kHz}$ and varies by $\sim 10 \mathrm{kHz}$ for nominally identical resonators, a final tuner adjustment range of at least $20-30 \mathrm{kHz}$ needs to be available when the system is cold. Finer tuning of a few hundred $\mathrm{Hz}$ is required to compensate for frequency shifts caused by changes in the rf coupler position and for slight changes in shape of the resonator due to changes in the helium pressure.

Several methods, all based on the controlled mechanical deformation of the cavity, have been successfully employed to compensate for frequency drifts on a time scale of seconds due to changes in the helium pressure. Some include stepping motors and compression screws while others use a pneumatic system operated with helium gas and a bellows to displace a portion of the cavity wall [3]. Also under development are a preloaded piezoelectric [4] or magnetorestrictive tuners [5]. Conventionally, a cavity with low stored energy is tuned capacitively by deflection of the tuning plate opposite the free ends of the loading arms. In the 1-QWR, the $1.0 \mathrm{~mm}$ thick end plate is deflected up to $1 \mathrm{~mm}$ for a tuning range of $20 \mathrm{kHz}$. The limitation of such a tuner is its small displacement range due to high stress in the structurally stiff tuner diaphragm. Since a high driving force is required, it is slow to respond, has poor resolution, and large backlash. This is less the case for bellows tuners.
Detuning of the resonant frequency on a millisecond time scale can be caused by microphonic noise or rapid fluctuations in helium delivery pressure which need to be compensated. For resonators with relatively low stored energy, $<0.07 \mathrm{~J} /(\mathrm{MV} / \mathrm{m})^{2}$ [6], the compensation is done by reducing the loaded $Q$ and overcoupling to a dynamic reactive quadrature control loop. For resonators with greater stored energy, a variable reactive load using a PIN diode network at the cavity wall provides the required $Q$-loading.

The 2- and 3-QWR design gradient is $\sim 5 \mathrm{MV} / \mathrm{m}$ (sputtered $\mathrm{Nb}$ coating) corresponding to a stored energy of $U_{0} \approx 1.2 \mathrm{~J}$ or $0.048 \mathrm{~J} /(\mathrm{MV} / \mathrm{m})^{2}$. The purely reactive tuning power $P_{f}$ is determined by $P_{f}=\pi U_{o} \Delta f_{1 / 2}$, where $\Delta f_{1 / 2}$ is the loaded half-bandwidth of the overcoupled system. The loaded bandwidth should be 6 times the microphonic noise plus twice the resolution of the me-

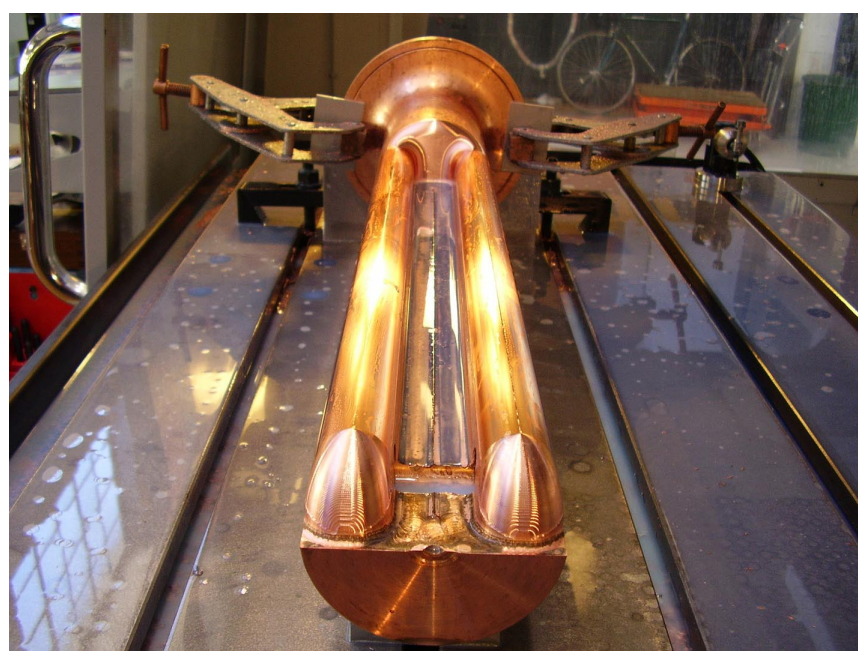

FIG. 1. (Color) The prototype 2-QWR halfway through the $\mathrm{CNC}$ machining process. 

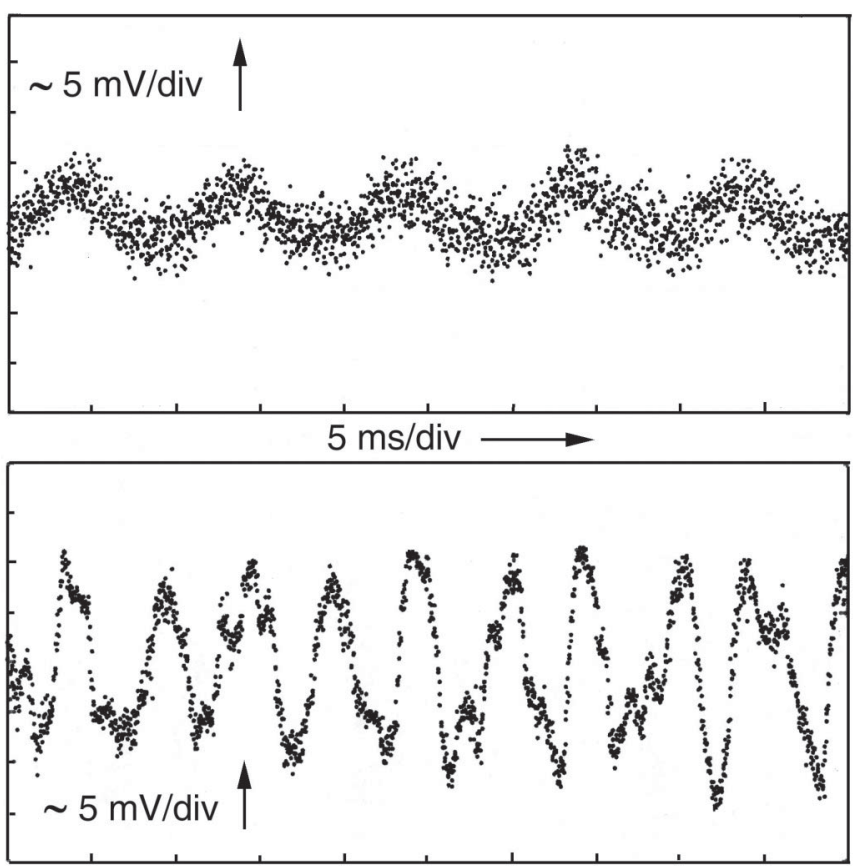

FIG. 2. Microphonic noise in an ANU LINAC cryostat. The top trace is the frequency error signal $(0.25 \mathrm{~Hz} / \mathrm{mV})$. The $\mathrm{rms}$ frequency background noise is about $1 \mathrm{~Hz}$. The bottom trace is the same signal while the slow mechanical tuner is operating. The rms frequency noise has increased to $2.3 \mathrm{~Hz}$.

chanical tuner [7]. The microphonic noise is expected to be below $3.5 \mathrm{~Hz} \mathrm{rms}$ based on the vibration performance of ANU LINAC cryostats shown in Fig. 2. The resonant frequency shaking in Fig. 2 was measured with a critically coupled split loop resonator running in self-excited loop mode at $3.5 \mathrm{MV} / \mathrm{m}$ with the amplifier delivering $6 \mathrm{~W}$. The top trace shows a frequency error corresponding to $0.25 \mathrm{~Hz} / \mathrm{mV}$ or about $1 \mathrm{~Hz} \mathrm{rms}$, due to background vibration noise. In the bottom trace the slow frequency tuner is being adjusted resulting in more than doubling of the rms frequency noise. This is still below the mechanical noise threshold limit of $3.5 \mathrm{~Hz}$ rms.

The sensitivity of the multistub cavity to helium pressure variations is $\Delta F / \Delta P \sim 0.01-0.02 \mathrm{~Hz} / \mathrm{mBar}$, similar to that of QWRs. At the ANU LINAC, the helium pressure is stabilized to within $\Delta P=10 \mathrm{mBar}$ [8]. Thus, this contribution is negligible compared to that caused by mechanical vibration. The multistub cavity fast tuner must be capable of achieving $\sim 1 \mathrm{~Hz}$, frequency resolution within a bandwidth of $\Delta f_{1 / 2}=22 \mathrm{~Hz}$. The required $P_{f}$ is $83 \mathrm{~W}$.

The design requirements for the slow tuner system for multistub resonators are given in Table I.

TABLE I. Multistub cavity tuner specifications.

\begin{tabular}{lc}
\hline \hline Frequency tuning range & $30 \mathrm{kHz}$ \\
Frequency resolution & $1 \mathrm{~Hz}$ \\
Cavity frequency at $4.3 \mathrm{~K}$ & $149984 \mathrm{kHz}$ \\
Cavity frequency at $300 \mathrm{~K}$ (air) & $149470 \mathrm{kHz}$ \\
\hline \hline
\end{tabular}

We report two complementary developments at ANU to achieve these design goals: first, a rotary tuner for the 2QWR and a displacement tuner for the 3-QWR. These tuners are only possible because a multistub resonator, in acceleration mode, has low currents between the outer cylinder and the tuner elements.

\section{ROTARY TUNER}

The rf current distribution and consequent power dissipation properties of the multistub cavities and their slow tuners were explored using Microwave Studio (MWS) electromagnetic analysis. Figure 3 shows the rf current in a conventional tuner plate of a $2-\mathrm{QWR}$, running in the accelerating mode, mode 2 . The opposite going currents are substantially confined to the plate and thus need not return through the rf joint. This is the first crucial feature of the 2-QWR that allows implementation of a rotary tuner instead of the conventional deflection plate. The other feature is that the mirror charge induced by the stub ends produces a current parallel to the beam direction. A rotary electrode will couple strongly to this current when its long axis is parallel to this direction and couple weakly when its axis is perpendicular to it. In the weak coupling configuration, the area of conductor near the ends of the stubs is reduced. This feature can be exploited for the 2-QWR since it affects both stubs equally. It is not suitable for the 3-QWR because it would mainly affect the outer stubs and so unbalance the fields between the stubs.

\section{A. Rotary tuner operation in nonaccelerating mode}

One can best appreciate the advantages of the rotary tuner for a $2-\mathrm{QWR}$ by contrasting it with a conventional

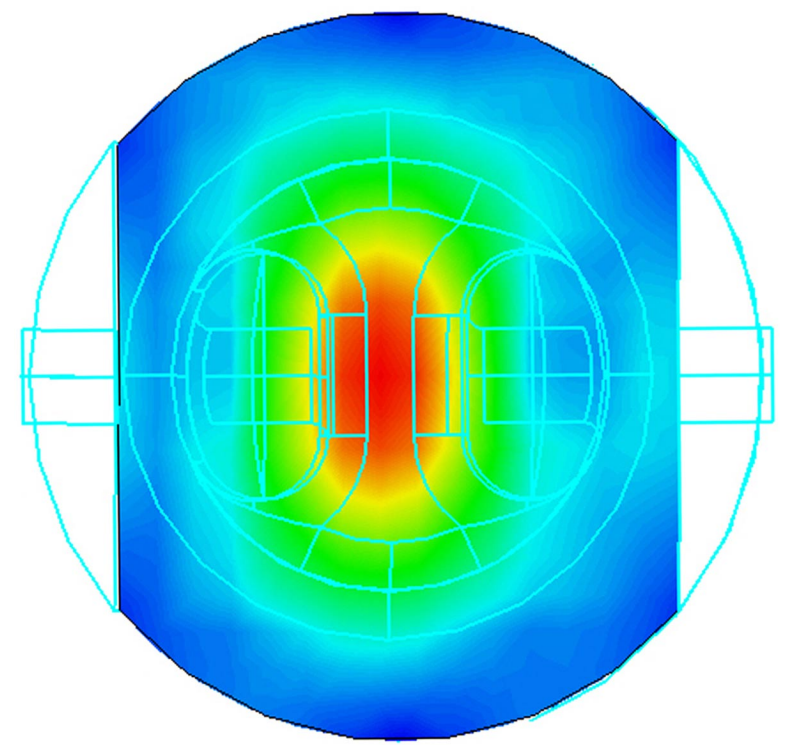

FIG. 3. (Color) Distribution of $H$-field, and thus the current in a conventional tuner plate of a 2-QWR operating in the accelerating mode. 


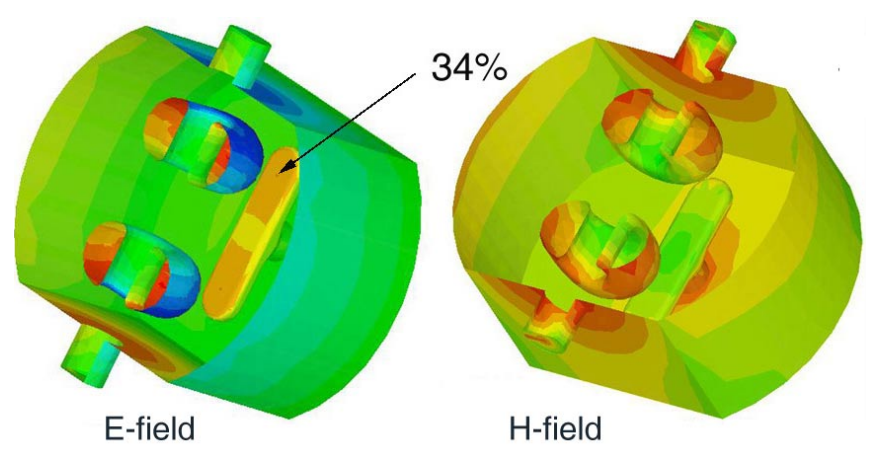

FIG. 4. (Color) Distribution of $E$-field and $H$-field at a rotary tuner (RT) in 2-QWR, mode 1 oriented along the beam axis. In the left picture, the number as a percentage is the ratio $E / E_{p}$, blue is negative charge and red positive charge. In the right picture, red corresponds to high $H$-field, green to low field.

tuner operating in a 1-QWR. This can be illustrated with the 2-QWR operating in the nonaccelerating mode, mode 1 , in which the current runs in parallel in each stub resulting in the same polarity on the ends of the stubs. Thus, in this mode, there is no electric field between the stubs but only between the stubs and the walls. This is analogous to the case of a conventional single stub, quarter-wave resonator.

Figure 4 shows the distribution of $E$-field and $H$-field in the rotary tuner electrode and its supporting plate in mode 1 for $30 \mathrm{~mm}$ between the tuner electrode and the loading elements. All rf currents have to go through the tuner shaft generating a substantial $H$-field around its base as is shown as red in Fig. 4 (right picture). The numbers in Fig. 4 display the ratios $E / E_{p}$ and $H / H_{p}$ in percent, where $E_{p}$ is the peak electric field and $H_{p}$ is the peak magnetic field in the resonator. For a rotary tuner with a $12 \mathrm{~mm}$ diameter shaft, the maximum of $H / H_{p}(7.7 \%)$ is on the shaft where it is attached to the base plate if the tuner bar is oriented along the beam axis. When the bar is oriented perpendicular to the beam axis $H / H_{p}$ is $5.7 \%$, since the capacitive coupling is considerably reduced in the latter position.

The maximum ratio $E / E_{p}$ on the surface of the tuner bar is $34 \%$ as shown in Fig. 4. On the surface of the support plate the maximum ratio $E / E_{p}$ is $1.7 \%$ since the support plate is shielded by the tuning bar and is $45 \mathrm{~mm}$ from the stub ends compared to the tuning bar distance of $30 \mathrm{~mm}$.

\section{B. Rotary tuner operation in accelerating mode 2}

A substantially different field distribution is obtained in the accelerating mode 2. Figure 5 shows the corresponding distribution of the $E$-field and the $H$-field. For this mode, the induced rf currents in the tuner bar need not all return through the tuner shaft and rotary joint. The numbers in Fig. 5 display the ratios $E / E_{p}$ and $H / H_{p}$ as a percentage where the maximum $\mathrm{H} / \mathrm{H}_{\mathrm{p}}$ field of $2 \%$ occurs in the center of the tuner bar. The maximum of $H / H_{p}$ on the shaft is $0.7 \%$ and occurs where it passes through the baseplate

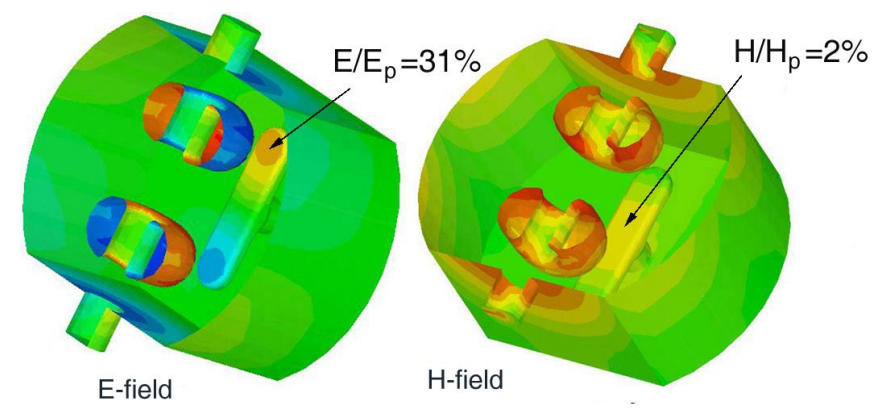

FIG. 5. (Color) Distribution of $E$-field and $H$-field at a RT, mode 2. Numbers display ratios $E / E_{p}$ and $H / H_{p}$ as a percentage. In the left picture, $E / E_{p}$, blue signifies negative charge and red positive charge. In the right picture, red corresponds to high $H$-field, green to low field.

when the tuner bar is oriented along beam axis. When the tuner bar is oriented perpendicular to the beam axis, $H / H_{p}$ is $0.5 \%$.

The maximum rf current around the base of the tuner shaft in mode 2 is about 11 times less than in mode 1 as a consequence of the fact that compensating currents are mainly confined to the tuner bar. A flexible connection will carry the rf current associated with this magnetic field on the shaft to the body of the resonator.

The power dissipation in the tuner bar was calculated using MWS software to estimate the cooling requirements for the bar. For the tuner bar aligned along beam axis, the power dissipated on the bar and its shaft surfaces is approximately $P=0.22 \mathrm{~mW}$ for $6 \mathrm{~W}$ absorbed in the cavity. Cooling and the rf current path for the bar are provided by a flexible lead-plated cooper braid $(l=30 \mathrm{~cm}$ and $A=$ $0.1 \mathrm{~cm}^{2}$ ) attached to the outer can at $4.3 \mathrm{~K}$, which limits the temperature rise to $17 \mathrm{mK}$.

\section{Mechanical design and performance}

Figure 6 shows the details of the rotary tuner mechanical design. The tuner consists of an $80 \times 20 \mathrm{~mm}$ flat bar with rounded edges (item 1) on a $20 \mathrm{~mm}$ diameter shaft (item 2) centered in a $24 \mathrm{~mm}$ diameter hole in the support plate through an insulating bearing (not shown). The tuner bar is rotated by an arc lever arm (item 3 ) driven by a springloaded, rotary shaft (item 4). The control range can be chosen by changing the tuner to stub end separation using inserts (items 5). The rotary shaft is turned by a long thin wall stainless steel tube attached to the ferrofluidic vacuum feedthrough, driven by a stepper motor. The drive shaft is slotted so that thermal contraction does not cause axial stress and small changes in direction of the drive axis are accommodated by antibacklash pivot bearings.

The small driving force required for the rotary tuner only needs a simple drive mechanism and so provides lowbacklash tuning. For even more precise tuning, a worm gear can be used instead of a friction drive. 


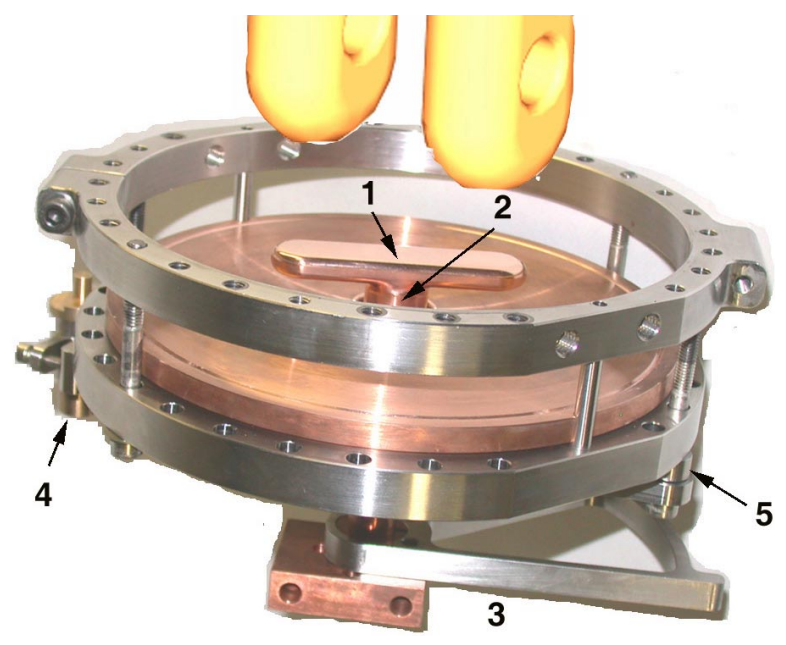

FIG. 6. (Color) Mechanical design of rotary tuner: 1-flat bar with rounded edges; 2-tuner shaft; 3-arc lever arm; 4driving rotary shaft; 5 - coarse tuning inserts.

Figure 7 shows the resonator frequency responses of a $20 \times 80 \mathrm{~mm}$ flat rotary tuner for different tuner-toloading elements separations, $l$. The minimum rf frequency occurs when the tuner capacitance is a maximum and the bar is aligned along the beam-axis-rotor angle of $0^{\circ}$. A $\pm 15 \mathrm{kHz}$ coarse range with $l=30 \mathrm{~mm}$ should be adequate to achieve the target frequency at $4.3 \mathrm{~K}$. For rotor angles of $30^{\circ}$ to $60^{\circ}$, the frequency gradient is about $0.5 \mathrm{kHz} /$ degree.

\section{Insertion loss}

The insertion loss (attenuation) is the reduction in transmitted signal power resulting from the insertion of a device (tuner) in a transmission network. The tuner must affect the resonator field to work but should not be so high as to compromise the overall performance. The impact of the turner would be revealed by a decrease in the forward

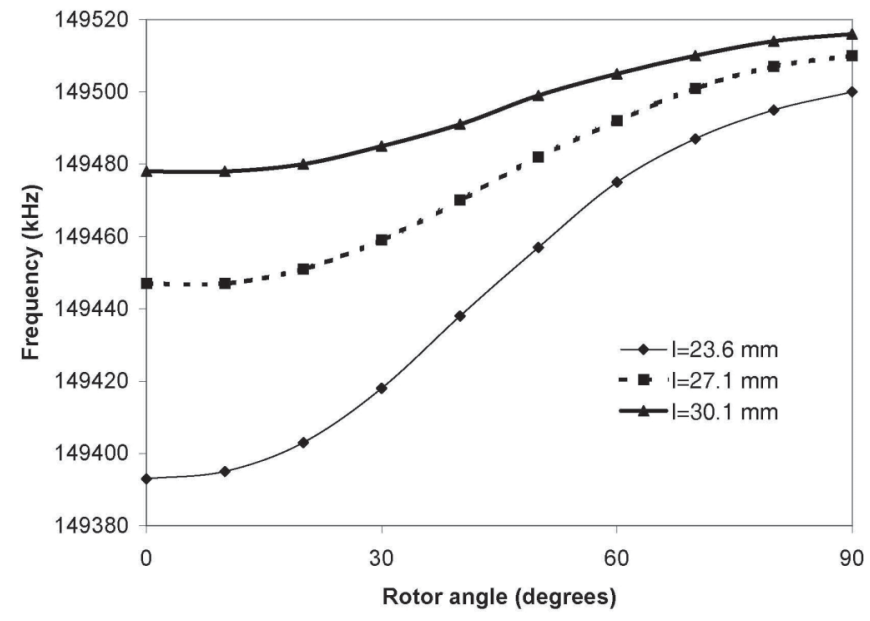

FIG. 7. Rotary tuner frequency ranges for 3 distances to the stub ends.

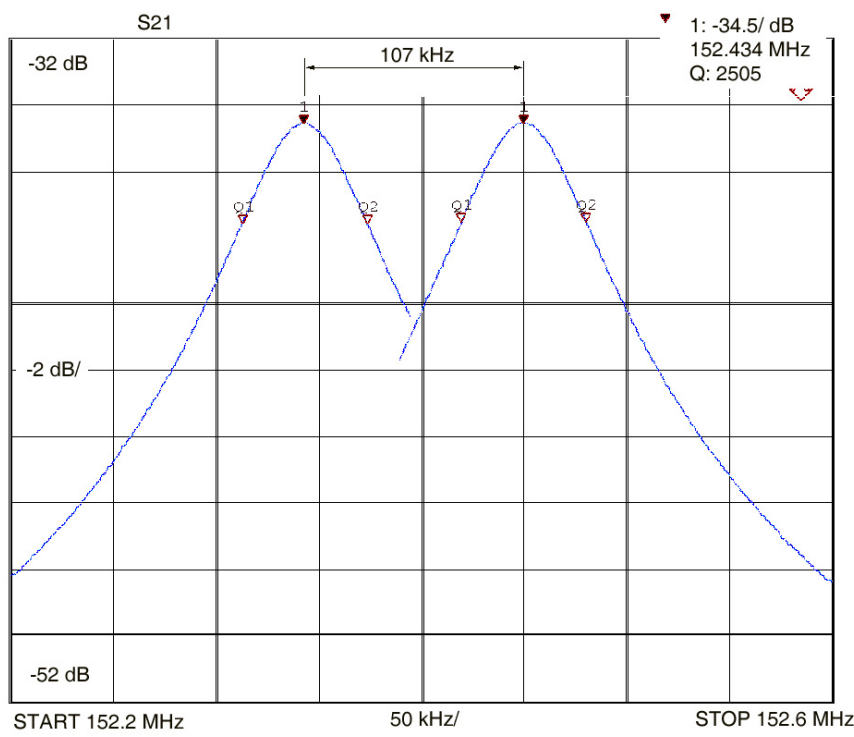

FIG. 8. (Color) Transmission characteristics $\mathrm{S}_{21}$ of the 2-QWR fitted with rotary tuner with $l=23.6 \mathrm{~mm}$ at the rotor angle of $0^{\circ}$ (left peak) and $90^{\circ}$ (right peak).

transmission coefficient $S_{21}$, the ratio of the output voltage from the capacitive pickup divided by the incident amplitude at the coupler. The value of $S_{21}$ of the resonator at room temperature is measured as a function of the tuner angle using a vector network analyzer and shown in Fig. 8.

Tuning characteristics were measured for a rotor angle from $0^{\circ}$ to $90^{\circ}$, at critical coupling. There is no noticeable change in the insertion loss over the range of frequency.

\section{DISPLACEMENT TUNER}

The simple rotary tuner however cannot be used for the 3-QWR because it would unbalance the electric fields between the stubs. Although one could employ a pair of rotary tuners - one at each centered on an outer sub gapthis would be cumbersome. A simpler alternative is a displacement tuner, which preserves the balanced $E$-field in all accelerating gaps. In this, the tuner bar is moved toward the stubs maintaining the same ratio of capacitive coupling to all loading elements. It still benefits from the low current in the shaft to the tuning electrode because that is an inherent feature of the multistub resonator concept.

As in the case of the 2-QWR appropriately $E$ - and $H$-field distributions are obtained for the accelerating mode, shown in Figs. 9 and 10. All data are presented for a gap of $20 \mathrm{~mm}$ between the displacement tuner and loading elements.

In this case too, the induced currents in the tuner bar itself, to a first approximation, need not return through the tuner shaft. The rf joint and therefore the $H$-field is not high in the plate supporting the bar as shown in Fig. 10. The numbers display the ratios $E / E_{p}$ and $H / H_{p}$ as a percentage. There is a maximum ratio $H / H_{p}$ of $0.6 \%$ on the base 


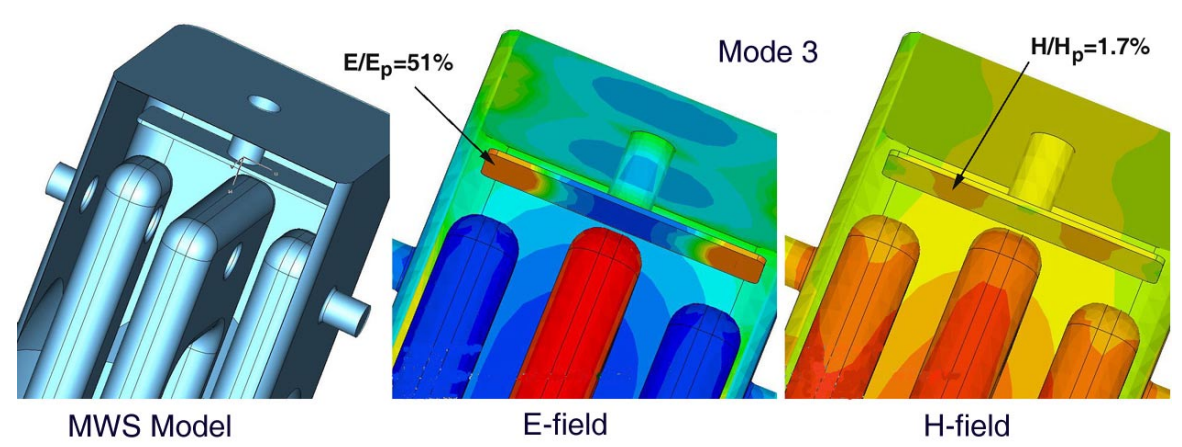

FIG. 9. (Color) Distribution of $E$-field, $H$-fields at a displacement bar tuner in a 3-QWR for the accelerating mode. The perspective of the figures makes the center stub appear to be closer to the tuner than the outer ones. In the center picture, $E / E_{p}$, blue signifies negative charge and red positive charge. In the rightmost picture, red corresponds to high $H$-field, green to low field.

of the shaft where it passes through the end plate when the tuner bar is positioned at its minimum distance from the stubs. As the bar is moved away from the stub ends along the $y$-axis, the tuning sensitivity is reduced as shown in Fig. 11. The maximum $E$-field on the surface of the tuner
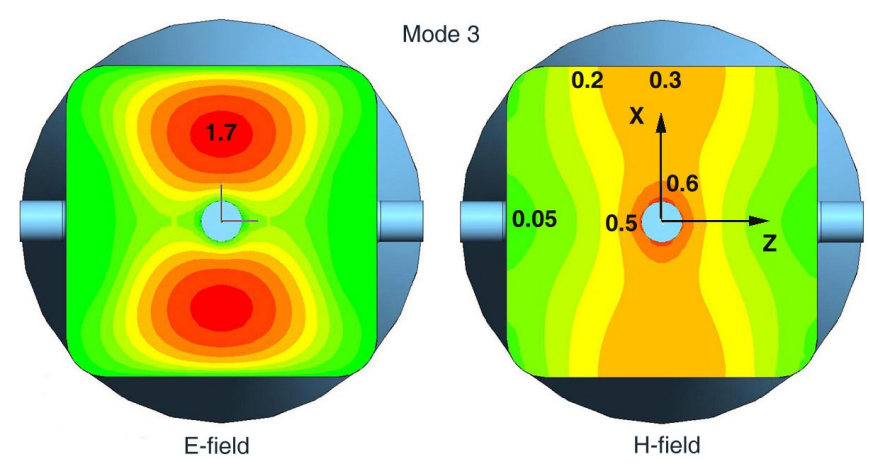

FIG. 10. (Color) Distribution of $E$-field, $H$-fields on the support plate of a displacement tuner. Numbers display ratios $E / E_{p}$ and $H / H_{p}$ as a percentage, for a distance of the bar to the stub ends of $20 \mathrm{~mm}$. Red corresponds to high field, green to low field.

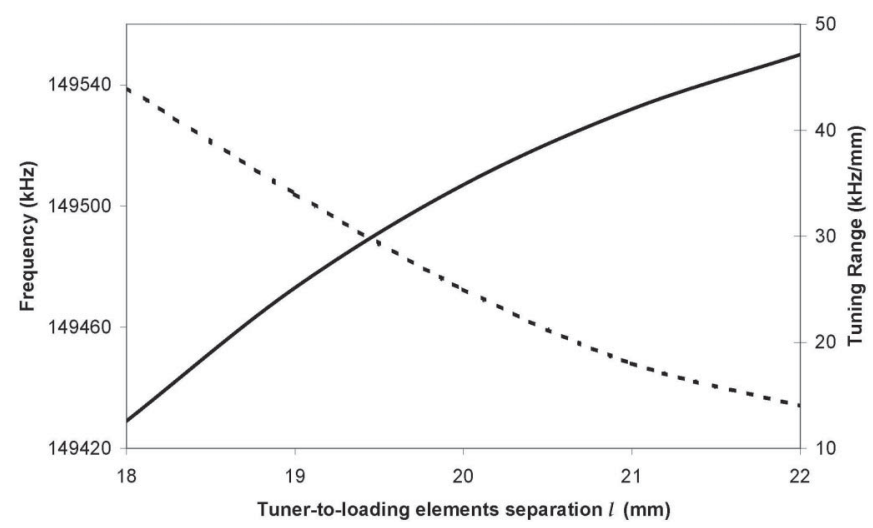

FIG. 11. Resonator frequency responses, calculated with MWS, of a $20 \times 100 \mathrm{~mm}$ flat displacement tuner (solid line) and tuning range (broken line) as a function of tuner-to-loading elements separation $l$. bar is $E / E_{p}=51 \%$, shown in Fig. 9, and $E / E_{p}$ is below $1.7 \%$ on the surface of the support plate.

The tuning range of $\Delta F / \Delta l=28 \mathrm{kHz} / \mathrm{mm}$ at a tunerto-loading elements separation $l=20 \mathrm{~mm}$ should be more than adequate to achieve the target frequency at $4.2 \mathrm{~K}$.

As in the rotary tuner, little current needs to flow from the tuning bar to the support plate. A length of lead-plated copper braid is sufficient to carry the residual current and to provide a cooling path. This is in contrast to 1-QWRs in which the larger tuning current must go this route and the more conductively robust and often stiffer connection may require substantial force to displace the tuning electrode.

The small driving force required for a displacement tuner in a multistub resonator allows low-backlash tuning. For precise tuning, a worm gear can be used to obtain a large step down between the control mechanics and the tuner shaft.

\section{CONCLUSION}

A credible design of rotary and displacement tuners for the 2-QWR and the 3-QWR cavities, respectively [1,2], have been developed and tested at room temperature. Future tests at $4.2 \mathrm{~K}$ will explore the adequacy of the $\mathrm{rf}$ and thermal connection from the tuning bar to the resonator body, the performance of the bearings, and frequency stability against vibration.

The multistub resonators intrinsically have low currents between the outer cylinder and the tuner elements. This allows the use of rotary or displacement tuners instead of the conventional deflection plate. The small driving force required for these tuners allows for low-backlash mechanisms. The use of the rotary tuner is limited to the resonators with two loading elements such as 2-QWR, conventional split loop resonator, and 2-stub, half wave resonators. The displacement tuner is more versatile and can be used for any TEM-like resonators with more than two loading elements.

The MWS calculations for the displacement tuner indicate an adequate tuning range up to few tens $\mathrm{kHz}$ with low insertion loss and sub-Hz precision. The frequency 
resolution of displacement tuners is expected to be somewhat larger than that of the rotary tuner and thus the rotary option is chosen for the 2-QWR.

The tuners are superior to mechanical devices presently used due to their high frequency precision and response bandwidth. In addition, the designs allow one to select the middle of the tuning range simply by inserting spacers, thus allowing more generous manufacturing tolerances for the geometry of the cavity. In addition, the new tuners have the desirable characteristics of wide tuning range, absence of sliding contacts, relatively high $Q$, and compact physical size.

The manufacturing of the $150 \mathrm{MHz} 2-\mathrm{QWR}$ with rotary tuner has been completed. The PbSn plating, exercised on the existing split loop resonators, will be extended to the 2QWR as a straightforward step to quickly explore the superconducting performance of the new geometry. The commissioning of the plating equipment has been started. In the longer term, the $\mathrm{Nb}$ sputter coating will be researched because, in principle, it can produce films competitive to the much more expensive solid niobium option and performs at higher fields than plated lead.

\section{ACKNOWLEDGMENTS}

The competence and skills of Alistair Muirhead in the use of Mechanical Desktop and the 2-QWR prototype design has been a key resource for the advancement of the project. The authors would like to thank mechanical workshop RSPhysSE ANU for their cooperation and enthusiasm during this work and skillful operation of the CNC machine to build a full-scale OHFC resonator. The authors thank Computer-Simulation Technology GmbH for providing an evaluation license for a package MWS to the Nuclear Physics Department.

[1] N. R. Lobanov and D.C. Weisser, Phys. Rev. ST Accel. Beams 9, 042002 (2006).

[2] N. R. Lobanov and D.C. Weisser, Phys. Rev. ST Accel. Beams 9, 112002 (2006).

[3] G.P. Zinkann, S. Sharamentov, and B. Clifft, in Proceedings of 2005 Particle Accelerator Conference, Knoxville, Tennessee, 2005, pp. 4090-4092.

[4] G. Devanz, P. Bosland, M. Desmons, E. Jacques, M. Luong, and B. Visentin, in Proceedings of EPAC 2006, Edinburgh, Scotland, 2007, pp. 378-380.

[5] C. Joshi, A. Mavanur, C.-Y. Tai, and T. Grimm, in Proceedings of Particle Accelerator Conference 2003, Portland, Oregon, pp. 1407-1409.

[6] G. P. Zinkann, in Proceedings of the 11th Workshop on rf Superconductivity, Travemunde, Germany, 2003.

[7] J. Delayen, RIA Driver Workshop II, Argonne, 2003.

[8] N. R. Lobanov and D.C. Weisser, in Proceedings of the Australian Institute of Physics, 16th Biennial Congress, Canberra, Australia, 2005. 